\title{
The Beck Depression Inventory for Primary Care accurately screened for major depressive disorders
}

\author{
Steer RA, Cavalieri TA, Leonard DM, et al. Use of the Beck Depression Inventory for Primary Care to screen for major depression \\ disorders. Gen Hosp Psychiatry 1999 Mar/Apr;21:106-11.
}

Question

Does the Beck Depression Inventory for Primary Care (BDI-PC) accurately screen for major depressive disorders in primary care patients?

\section{Design}

Blinded comparison of the results of the BDI-PC with diagnosis based on the Diagnostic and Statistical Manual of Mental Disorders, fourth edition (DSM-IV) criteria obtained using the Mood Module from the Primary Care Evaluation of Mental Disorders (MM from the PRIME-MD).

\section{Setting}

A university based outpatient clinical centre in Stratford, New Jersey, USA.

\section{Patients}

120 patients (mean age 58 y, $50 \%$ men, $86 \%$ white) who were scheduled for a consecutive routine outpatient visit with a physician specialising in internal medicine.

\section{Description of test and diagnostic standard}

Patients completed the BDI-PC in the waiting area before seeing the physician. The BDI-PC is a 7 item questionnaire with each item rated on a 4 point scale (0-3). It is scored by summing ratings for each item (range 0-21). Items are symptoms of sadness, pessimism, past failure, loss of pleasure, self dislike, self criticalness, and suicidal thoughts and wishes. When completing the questionnaire, patients were asked to describe their symptoms for the "past 2 weeks including today." The diagnostic standard, an interview guided by the MM from the PRIME-MD using $D S M-I V$ criteria, was administered by a physician who was blinded to the results of the BDI-PC.

\section{Main outcome measures}

Sensitivity, specificity, likelihood ratios for positive and negative results, and area under the receiver operating characteristic (ROC) curve.

\section{Main results}

29 patients (24\%) were given a diagnosis of major depressive disorder on the basis of the $D S M-I V$ criteria from the interview. The mean BDI-PC score differed for the 29 patients diagnosed with major depressive disorder compared with the 91 without (6.55 v $0.79, \mathrm{p}<0.001)$. At a $\geq 4$ cutoff score, the BDI-PC had a sensitivity of $97 \%$ (95\% CI $82 \%$ to $99 \%$ ), a specificity of $99 \%$ (CI $94 \%$ to $99 \%$ ), and \{likelihood ratios for a positive and negative result of 97 and 0.03 , respectively $\}^{*}$. The area under the ROC curve for the BDI-PC was 0.99 (SE 0.01), showing that the BDI-PC had a very high level of differentiation (area under the ROC curve of 0.50 indicates chance discrimination). BDI-PC score was not related to patient sex, race, age, total number of medical disorders, or whether an internal medicine specialist was being seen.

\section{Conclusion}

The Beck Depression Inventory for Primary Care was an accurate test for screening primary care patients for major depressive disorders.

*Numbers calculated from data in article.

Source of funding: no external funding.

For correspondence: $\operatorname{Dr}$ R A Steer, University of Medicine and Dentistry of New Jersey, School of Osteopathic Medicine, Department of Psychiatry, 40 East Laurel, PCC 218, Stratford, NJ 08084-1350, USA.Fax +1 6095666030 .

\section{Commentary}

The theoretical basis for this study is the knowledge that clinical depression in both primary care settings and in patients with chronic and life threatening illnesses is far more common than earlier epidemiological studies suggest. The authors acknowledge that clinical depression is under diagnosed and is a quality of life issue. An accurate tool is therefore needed for screening these patients for depressive symptoms.

This study of the BDI-PC by Steer et al is methodologically sound. It includes a sufficient number of patients; the physicians who diagnosed patients with depression were unaware of the patients' answers to the BDI-PC and therefore not influenced by their scores; and analyses were conducted to ensure that the BDI-PC scores were not significantly correlated with sex, age, race, total number of medical disorders, or treatment by a generalist or specialist.
The results support the BDI-PC as an accurate and effective screening tool. This finding is consistent with previous research that found high sensitivity and specificity rates when using the BDI-PC to identify medical inpatients and adolescents with and without major depressive disorders. ${ }^{1}{ }^{2}$

The BDI-PC will be useful to hospital and community nurses and health visitors who come into contact with, and often have long term and trusting relationships with, patients who have chronic and life threatening conditions and their families. These healthcare workers can now be confident that they have a credible and workable tool to identify major depressive disorders. This study also has relevance for mental health nurses who are often involved in supporting and advising nonspecialist nurses in their assessment of patients with suspected depression. The tool is easy to use, workable in practice, and gives an objective perspective, which will enhance nurses' global assessment of their patients.

Although this tool is effective for use in a primary care setting, it is important that it be used as a screening tool rather than as a diagnostic tool. Patients with a BDI-PC score $>4$ should be referred for a more detailed psychiatric assessment, which will enable, if needed, early and expert intervention from a mental health specialist.

Catherine Bellis, RGN, RMN Clinical Team Leader, Old Age Psychiatry Hinchingbrooke Health Care NHS Trust Huntingdon, UK

1 Beck AT, Guth D, Steer RA, et al. Screening for major depression disorders in medical inpatients with the Beck depression inventory for primary care Behav Res Ther 1997:35:785-91. 2 Wrimary care. Behav Res Ther 1997;35:785-91. Screening, Steer RA, Jones-Hicks L, et al. Screening for major depression disorders in atpatients with Beck depression inventory for primary care.J Adolesc Health 1999:24:389-94 